\title{
Retos y oportunidades en la enseñanza de las matemáticas en el bachillerato de la UAA
}

Efraín Macias Hernández

La esencia de las matemáticas no es hacer las cosas simples complicadas, sino hacer las cosas complicadas simples.

S. Gudder

Resumen

$\mathrm{E}$ nseñar implica tomar en consideración tanto a los actores del proceso educativo como el contexto cultural y social en el que está inserto este proceso; dicho contexto se determina o delinea en un currículum o plan de estudios del cual se desprenden los diferentes programas de materia. Enseñar matemáticas, específicamente a nivel medio superior, implica también reconocer aspectos, tales como los conocimientos con los que egresan los estudiantes de educación básica y su actitud ante esta asignatura. El propósito del presente artículo es propiciar una reflexión en torno al quehacer del docente de matemáticas en este nivel educativo y las estrategias que se pueden incorporar en el aula para enfrentar el reto de enseñar cualquier materia de esta área del conocimiento.

Palabras clave: matemáticas, enseñanza, bachillerato, retos, oportunidades.

\section{Introducción}

Tradicionalmente, cualquier materia del área de la matemática ha sido considerada por los estudiantes de los diferentes niveles educativos como "difícil" por antonomasia. El problema se agrava cuando esta percepción es reafirmada por los mismos profesores que, por diversos motivos, muchas veces inconscientemente la presentan de esta manera a sus alumnos. Es así que se va generando una idea equivocada de lo que son las matemáticas en las mentes de los educandos desde los primeros años de su formación académica, de manera que las consideran, además de difíciles, abstractas, sin conexión con el mundo real y sin alguna utilidad práctica (Gómez, 2002). Son muchos los retos que debe enfrentar un profesor que imparte cualquier materia del área de las matemáticas si desea que sus estudiantes aprendan y cambien su percepción frente a estas asignaturas. En este sentido, parece adecuado reflexionar en torno a ciertos elementos, como por ejemplo el contexto educativo en el que se desempeñan los profesores, la información que proveen los diferentes exámenes, tanto diagnósticos como de egreso, y la posibilidad de utilizar algunos recursos con el fin de mejorar la enseñanza y, por lo tanto, el aprendizaje de las matemáticas.

\section{La comprensión de la educación en el Modelo Educativo Institucional}

Un referente para abordar el contexto educativo es el Modelo Educativo Institucional (MEI) de la Universidad Autónoma de Aguascalientes (UAA), en el que se concibe el proceso educativo como "intencional y sistemático" y, además, "combina de forma armónica y equilibrada [...]; lo universal y lo singular; la tradición y la modernidad" (UAA, 2007: 6). Si desde la docencia se entiende esto, es fácil ver los alcances y condiciones que se requieren en esta labor. Así, es deseable que una educación de calidad se caracterice, entre otras, por ser equitativa, pertinente, innovadora, responsable y, sobre todo, estar enfocada al estudiante y a su aprendizaje. Del MEI se rescatan también las tareas y rasgos de los actores educativos: estudiantes y profesores; para estos últimos, el documento en cuestión menciona que sean:

Capaces de asumir los distintos roles que la tarea docente requiere $[\ldots]$, profesionales comprometidos con la Universidad, con la excelencia académica, con las metas educativas y con su entorno social [y ser], profesionales que dominan sus áreas de conocimiento, lo que les permite 
manejar con fluidez los contenidos de aprendizaje, además de que cuentan con conocimiento pedagógico, curricular, didáctico y del contexto social (UAA, 2007: 9-10).

Todas estas características habilitan al profesor de matemáticas para hacerle frente a los retos que surgen en el día a día de su labor docente.

\section{Retos en la enseñanza de las matemáticas}

Los docentes que imparten alguna materia del área de la matemática a nivel bachillerato se enfrentan a ciertos retos relacionados con los estudiantes, como una actitud de rechazo o poca motivación hacia la materia desde la formación básica, bases poco sólidas de los contenidos y una excesiva dependencia de la tecnología (Ricoy y Couto, 2018; Cárdenas, 2017).

El informe del Instituto Nacional para la Evaluación de la Educación (INEE, 2018) "La educación obligatoria en México", coordinado por Schmelkes y Zorrilla (2018), presenta un histórico de los resultados en las evaluaciones en lenguaje y comunicación y matemáticas desde preescolar hasta educación media superior de 2005 a 2017; con resultados de las pruebas EXCALE, PLANEA, ENLACE y PISA, ofreciendo una panorámica de la educación en México en estos dos rubros. En el capítulo siete de dicho informe se abordan algunos aspectos relevantes que se deben considerar al momento de hablar de la enseñanza de las matemáticas.

En primer lugar, el informe refiere únicamente datos de las áreas de lenguaje y comunicación y matemáticas porque: "Son áreas que implican el desarrollo de habilidades verbales (lectura, escritura y oralidad) y matemáticas (pensamiento analítico, sintético y resolución lógica de problemas), indispensables para adquirir nuevos conocimientos, aplicarlos y transformarlos" (p. 269), reconociendo la importancia del dominio que deben tener los estudiantes en estas disciplinas.

En un segundo término, el informe alude a los conocimientos y habilidades que se esperaría los estudiantes desarrollasen durante la educación obligatoria en nuestro país, como: "argumentar y estructurar su razonamiento, propiciar el pensamiento reflexivo, lógico y crítico; construir e interpretar situaciones reales, hipotéticas y formales; formular, resolver y argumentar la solución de problemas por medio de diferentes métodos y procedimientos numéricos, algebraicos, gráficos, geométricos y estadísticos" (p. 275).

En tercer lugar, y específicamente concerniente a este artículo, el informe establece aquellas habilidades y conocimientos matemáticos que los estudiantes egresados de secundaria deberían tener, los mismos requeridos para acceder con éxito al bachillerato. No obstante, los resultados de los exámenes indican que de manera reiterada la mayoría de los estudiantes de los diferentes niveles educativos se ubican en el nivel de logro más bajo, lo cual implica una preparación deficiente de gran parte de los estudiantes desde preescolar hasta el término de su educación básica (al egresar de secundaria, 65\% se ubica en este nivel de logro). Y añade el informe que la situación no mejora en la educación media superior, pues $66 \%$ de los estudiantes se encuentra en el nivel I (NI), el más bajo de ejecución, como se muestra en la Tabla 1:

Tabla 1. Resultados nacionales de aprendizaje en matemáticas en diferentes grados de la educación obligatoria

\begin{tabular}{lccccccc}
\hline Grado & $\begin{array}{c}\text { Año de } \\
\text { aplicación }\end{array}$ & $\begin{array}{c}\text { Media } \\
\text { nacional }\end{array}$ & NI & NII & NIII & NIV & $\begin{array}{c}\text { Grupos desfavorecidos } \\
\text { (y diferencia entre el promedio } \\
\text { nacional y el promedio de los grupos) }\end{array}$ \\
\hline Tercero de preescolar & 2011 & 497 & 9 & 50 & 27 & 14 & $\begin{array}{l}\text { Cursos comunitarios (45) } \\
\text { Preescolares generales rurales (26) }\end{array}$ \\
\hline Tercero de primaria & 2014 & 519 & 19 & 37 & 31 & 13 & Escuelas indígenas (33) \\
\hline Sexto de primaria & 2015 & 500 & 61 & 19 & 14 & 7 & $\begin{array}{c}\text { Escuelas indígenas (62) } \\
\text { Cursos comunitarios (22) }\end{array}$ \\
\hline Tercero de secundaria & 2017 & 497 & 65 & 22 & 9 & 5 & $\begin{array}{l}\text { Secundaria comunitaria (81) } \\
\text { Telesecundaria (22) }\end{array}$ \\
\hline
\end{tabular}




\begin{tabular}{|c|c|c|c|c|c|c|c|}
\hline Grado & $\begin{array}{c}\text { Año de } \\
\text { aplicación }\end{array}$ & $\begin{array}{c}\text { Media } \\
\text { nacional }\end{array}$ & NI & NII & NIII & NIV & $\begin{array}{c}\text { Grupos desfavorecidos } \\
\text { (y diferencia entre el promedio } \\
\text { nacional y el promedio de los grupos) }\end{array}$ \\
\hline $\begin{array}{l}\text { Último grado de media } \\
\text { superior }\end{array}$ & 2017 & 500 & 66 & 23 & 8 & 3 & $\begin{array}{l}\text { Telebachillerato comunitario ( } 37) \text {, } \\
\text { CONALEP (26), Telebachillerato ( } 24) \text {, } \\
\text { DGETA (19), Bachillerato Estatal DGE-CGE* } \\
(18)\end{array}$ \\
\hline
\end{tabular}

Fuente: Schmelkes y Zorrilla (2018: 277).

El informe en cuestión también establece lo que se espera de los egresados de bachillerato en el área de matemáticas:

En EMS se espera que los estudiantes apliquen el conocimiento matemático para resolver problemas, argumenten sus estrategias de solución e interpreten los resultados. Empleen diferentes enfoques y procedimientos matemáticos: aritméticos, geométricos, gráficos, algebraicos y tabulares, entre otros. Que construyan e interpreten modelos matemáticos de procesos sociales o naturales que sirvan para estudiar su comportamiento. Cuantifiquen, representen y comparen, experimental o matemáticamente, magnitudes y propiedades físicas de los objetos, y elijan un enfoque determinista o uno aleatorio para el estudio de un proceso o fenómeno (p. 276).

Esto nos da un panorama de lo que enfrenta el profesor de matemáticas en el bachillerato. Por una parte, recibe estudiantes que arrastran una cadena de deficiencias con las que debe lidiar en las diferentes asignaturas, y por otra, los conocimientos y las habilidades que debe fomentar en los estudiantes a su paso por el bachillerato.

\section{Oportunidades}

Un buen desempeño del docente de matemáticas en el nivel medio superior, que como consecuencia tenga mayor incidencia en el aprendizaje de los estudiantes, implica una serie de conocimientos, habilidades y actitudes necesarios para el logro de los objetivos. En concreto, del MEI (UAA, 2007) y de algunos autores como Gómez (2002), Rico y Lupiáñez (2008), Minerva (2002) y Cárdenas (2017), se rescatan:

1. Conocimiento del marco curricular. Rico y Lupiáñez (2008) señalan la importancia de conocer el marco curricular, ya que de ahí se derivan algunas cuestiones que el profesor debe responder al momento de contextualizar el aprendizaje, tales como: “¿qué transmitir?”, “¿qué características relevantes diferencian este conocimiento (el matemático) de otros?”, “¿por qué es importante este conocimiento para la educación?”, “¿cómo se produce el aprendizaje?”, “¿cómo aprenden los jóvenes?” (p. 35).

2. Conocimientos sólidos de las materias que imparte. Es indispensable que el profesor tenga dominio de los contenidos de las diferentes materias de esta área, dado que es lo que legitima su labor frente al grupo.

3. Incorporación de los recursos multimedia en las clases. En la actualidad, el uso de las tecnologías de la información y la comunicación (laptops, tablets, celulares) está inmerso en todos los espacios de la vida, y el educativo no es la excepción. En la medida en que los docentes encuentren y/o diseñen estrategias para utilizar en sus clases estas tecnologías, y no solamente gis y pizarrón, los estudiantes tendrán la oportunidad de valorar su uso (Gómez, 2002: 125).

4. Indagación y estructuración de materias en contexto, buscando aplicaciones prácticas de los temas del programa. Desarrollar una clase de matemáticas teniendo presente la capacidad de aplicar los conocimientos matemáticos a la vida cotidiana es una característica fundamental y deseable del proceso educativo en los modelos por competencias, mediante el diseño de experiencias de aprendizaje (UAA, 2007: 12; Gómez, 2002: 130).

« Un buen desempeño del docente de matemáticas en el nivel medio superior implica una serie de conocimientos, habilidades y actitudes necesarios para el logro de los objetivos. 》 


\section{4}

DOQERE Retos y oportunidades en la enseñanza de las matemáticas en el bachillerato de la UAA

5. Actualización permanente en el uso de las tecnologías aplicadas a la didáctica de las matemáticas (software para graficar, por ejemplo). Otro aspecto a tomar en cuenta es la posibilidad que brindan muchos programas educativos de software (algunos de ellos disponibles de manera gratuita y muy amigables en su manejo) para hacer más accesibles tales o cuales contenidos matemáticos. Como un ejemplo se puede mencionar el graficador Winplot, que se puede utilizar desde álgebra hasta cálculo diferencial e integral.

6. Búsqueda y/o creación y adecuación de materiales didácticos para las diferentes asignaturas. Utilizar juegos de mesa, acertijos y juegos mentales para introducir temas particulares, con el objetivo de afirmarlos o simplemente como una estrategia didáctica que le permita al estudiante aprender dichos temas en un contexto que, además, fomente otros aspectos importantes como su creatividad y el trabajo en equipo, pueden resultar en un impacto positivo en el aprendizaje de los contenidos (Minerva, 2002; Cárdenas, 2017).

\section{Conclusiones}

La enseñanza de las matemáticas en el nivel medio superior puede resultar un reto para los profesores de hoy. Las condiciones académicas con las que ingresan los alumnos en la disciplina que se desea enseñar y los resultados obtenidos en las evaluaciones de egreso de los jóvenes bachilleres, tanto nacionales como internacionales en el área de matemáticas, presentan un panorama inquietante $\mathrm{y}$, al mismo tiempo, un reto en su formación, actualización y desempeño. Su papel se percibe relevante en el proceso de enseñanza-aprendizaje, por lo cual es necesario que el profesor encuentre en los materiales y recursos disponibles una oportunidad para lograr atraer la atención de los alumnos y facilitar mejores aprendizajes en las diferentes asignaturas del área de las matemáticas. En otras palabras, es necesario que el docente asuma un proceso de mejora continua por medio de una autoconstrucción y autocrítica constante sobre la propia labor, acompañada de una actualización permanente en los recursos y materiales que sean pertinentes a las materias que imparte, incorporándolos paulatinamente en las clases y tendiendo a hacer del aula un espacio en el que los estudiantes se reencuentren con la belleza y utilidad de las matemáticas.

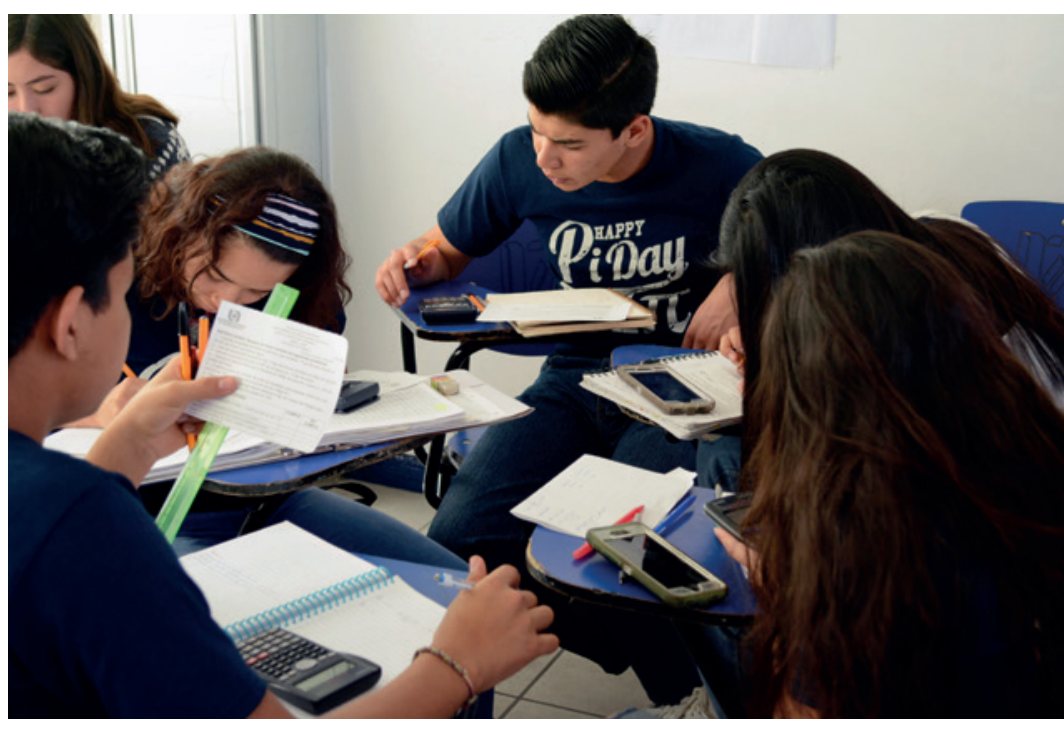

Fuentes de consulta

Cárdenas, W. (2017). Estrategias didácticas de aprendizaje en matemáticas. Consultado el 24 de noviembre de 2018 en: https://repository.unimilitar.edu.co/bitstream/handle/10654/16136/ C\%E1rdenasRodriguezWilliam2017.pdf;jsession id=30B65133250BCB09CCF601F43A54BD31? sequence $=1$.

Gómez, J. (2002). De la enseñanza al aprendizaje de las matemáticas. España: Paidós.

Minerva, C. (2002). El juego: una estrategia importante. EDUCERE, 6(19), 289-296. Venezuela: Universidad de los Andes. Consultado el 8 de marzo de 2019 en: https://www.redalyc.org/articulo.oa?id=35601907.

Rico, L. y Lupiáñez, J. (2008). Competencias matemáticas desde una perspectiva curricular. España: Alianza Editorial.

Ricoy, M. y Couto, M. (2018). Desmotivación del alumnado de secundaria en la materia de matemáticas. Revista Electrónica de Investigación Educativa, 20(3), 69-79. Recuperado en: https:// doi.org/10.24320/redie.2018.20.3.1650.

Schmelkes, S. y Zorrilla, M. (Coords.). (2018). La educación obligatoria en México. Informe 2018. México: Instituto Nacional para la Evaluación de la Educación.

UAA (2007). Modelo Educativo Institucional. Correo Universitario, séptima época, (15). [Primera reimpresión] 29 de mayo de 2015. México: Universidad Autónoma de Aguascalientes. Recuperado en: https://bit.ly/2OrlYxX. 